\title{
BMI open Home telemonitoring study for Japanese patients with heart failure (HOMES-HF): protocol for a multicentre randomised controlled trial
}

\author{
Norihiko Kotooka, ${ }^{1}$ Machiko Asaka, ${ }^{1}$ Yasunori Sato, ${ }^{2}$ Yoshiharu Kinugasa, ${ }^{3}$ \\ Kotaro Nochioka, ${ }^{4}$ Atsushi Mizuno, ${ }^{5}$ Daisuke Nagatomo, ${ }^{1}$ Daigo Mine, ${ }^{6}$ \\ Yoko Yamada, ${ }^{7}$ Kazuo Eguchi, ${ }^{8}$ Hideki Hanaoka, ${ }^{2}$ Takayuki Inomata, ${ }^{9}$ \\ Yoshihiro Fukumoto, ${ }^{4}$ Kazuhiro Yamamoto, ${ }^{3}$ Hiroyuki Tsutsui, ${ }^{10}$ \\ Tohru Masuyama, ${ }^{11}$ Masafumi Kitakaze, ${ }^{12}$ Teruo Inoue, ${ }^{13}$ Hiroaki Shimokawa, ${ }^{4}$ \\ Shin-ichi Momomura, ${ }^{7}$ Yoshihiko Seino, ${ }^{14}$ Koichi Node, ${ }^{1}$ on behalf of the \\ HOMES-HF study investigators
}

To cite: Kotooka N, Asaka M, Sato $\mathrm{Y}$, et al. Home telemonitoring study for Japanese patients with heart failure (HOMES-HF): protocol for a multicentre randomised controlled trial. BMJ Open 2013:3:e002972.

doi:10.1136/bmjopen-2013002972

- Prepublication history and additional material for this paper is available online. To view these files please visit the journal online (http://dx.doi.org/10.1136/ bmjopen-2013-002972).

Received 29 March 2013 Revised 19 April 2013 Accepted 22 April 2013

This final article is available for use under the terms of the Creative Commons Attribution Non-Commercial 2.0 Licence; see http://bmjopen.bmj.com

For numbered affiliations see end of article.

Correspondence to Dr Norihiko Kotooka; kotooka@cc.saga-u.ac.jp

\section{ABSTRACT}

Introduction: Despite the encouraging results from several randomised controlled trials (RCTs) and metaanalyses, the ability of home telemonitoring for heart failure (HF) to improve patient outcomes remains controversial as a consequence of the two recent largescale RCTs. However, it has been suggested that there is a subgroup of patients with HF who may benefit from telemonitoring. The aim of the present study was to investigate whether an HF management programme using telemonitoring could improve outcomes in patients with HF under the Japanese healthcare system. Methods and analysis: The Home Telemonitoring Study for Japanese Patients with Heart Failure (HOMES-HF) study is a prospective, multicentre RCT to investigate the effectiveness of home telemonitoring on the primary composite endpoint of all-cause death and rehospitalisation due to worsening HF in recently admitted HF patients (aged 20 and older, New York Heart Association classes II-III). The telemonitoring system is an automated physiological monitoring system including body weight, blood pressure and pulse rate by full-time nurses 7 days a week. Additionally, the system was designed to make it a high priority to support patient's self-care instead of an early detection of HF decompensation. A total sample size of 420 patients is planned according to the Schoenfeld and Richter method. Eligible patients are randomly assigned via a website to either the telemonitoring group or the usual care group by using a minimisation method with biased-coin assignment balancing on age, left ventricular ejection fraction and a history of ischaemic heart disease. Participants will be enrolled until August 2013 and followed until August 2014. Time to events will be estimated using the Kaplan-Meier method, and HRs and 95\% Cls will be calculated using the Cox proportional hazards models with stratification factors.

Trial Registration: The study is registered at UMIN Clinical Trials Registry (UMIN000006839).

\section{ARTICLE SUMMARY}

Article focus

- This study focuses on a role of the home telemonitoring system for patients with chronic heart failure (HF) to reduce hospital readmission under the Japanese healthcare system.

\section{Key messages}

- The ability of home telemonitoring for HF care to improve patient outcomes remains controversial. However, recent studies have suggested the existence of a subgroup of patients who might be able to benefit from the telemonitoring.

- The Home Telemonitoring Study for Japanese Patients with Heart Failure (HOMES-HF) study was specially designed for the participants and healthcare professionals to maintain adherence to daily measurement of body weight and blood pressure, to enhance clinician-patient communication and to empower their self-management by introducing a concept based on the idea of patient-centered care into the telemonitoring system.

Strengths and limitations of this study

- The HOMES-HF study will be the first trial of home telemonitoring for Japanese patients with HF.

- Multidisciplinary HF management systems have been underdeveloped and there are still no practicable telemonitoring systems for HF management operated by either the public or private sector in Japan. Therefore, devices using the HOMES-HF study are not designed exclusively for HF management; rather, they are based on a commercial-based health-maintenance product and customised for the study.

- The responsibility for acting on the information from the telemonitoring centre rests with each patient's physician; therefore, treatment will vary with each physician and institute. 


\section{INTRODUCTION}

Heart failure (HF) is one of the most common causes of hospital admission in developed countries. Hospital discharges of HF increased from 399000 in 1979 to 1099000 in 2004 in the USA. Moreover, of the $\$ 33.2$ billion in overall costs for HF care in 2007, $\$ 17.8$ billion was spent on in-hospital care. ${ }^{1}$ Available data for Japanese patients with HF indicate that approximately $70 \%$ of HF patients are older than 65 years of age and that about $35 \%$ of patients are readmitted for acute HF decompensation within 1 year of hospital discharge..$^{2-5}$ Owing to the rapid ageing of the population, there are growing concerns about the increased incidence and prevalence of $\mathrm{HF}$, and the high readmission rates and medical costs of hospitalisation have become a growing burden on the healthcare system.

Multidisciplinary HF management programmes and home-based care might be able to reduce hospital readmissions due to worsening $\mathrm{HF}^{6-9}$ Telemonitoring has grown to have a place in the HF disease management programmes. Chaudhry et $a l^{10}$ have expounded on the details of telemonitoring, categorising it into three groups with regard to the types of intervention, including telephone-based symptom monitoring, automated monitoring of signs and symptoms and automated physiological monitoring.

Recent meta-analyses and comprehensive reviews of several randomised controlled trials (RCTs) and cohort studies have shown that home telemonitoring as an adjunct to usual care reduces HF-related hospitalisations. In some trials, home telemonitoring has been found to reduce all-cause mortality and improve health-related quality of life. ${ }^{10-14}$ In contrast, recently published, welldesigned, large, prospective, multicentre RCTs have ended with disappointing results. In the Telemonitoring to Improve Heart Failure Outcomes (Tele-HF) trial, there were no significant differences between the automated monitoring of the signs and symptoms group and the usual care group with regard to the primary composite endpoint of all-cause readmission and death or the secondary endpoints including $\mathrm{HF}$ readmission. ${ }^{15}$ Although there has been criticism concerning patient adherence in the Tele-HF study, the incidences of the primary endpoint of all-cause mortality and the secondary endpoints of the composite of cardiovascular death and HF hospitalisation did not differ between the automated physiological monitoring group and the usual care group in the Telemedical Interventional Monitoring in Heart Failure (TIM-HF) study; nevertheless, the patient adherence to the telemonitoring intervention was acceptable. ${ }^{16}$ Therefore, the ability of home telemonitoring for HF care to improve patient outcomes remains controversial. ${ }^{17} 18$ The prospectively defined subgroup analysis of the TIM-HF study, which attempted to identify patients who may potentially benefit from telemonitoring, showed that treatment was significantly effective in the subgroup of patients with a prior history of HF decompensation, implantable cardiac defibrillator/cardioverter (ICD) implants or Patient Health Questionnaire (PHQ-9) scores of $<10$ for outcome days lost due to HF hospitalisation or death. ${ }^{19}$

\section{AIMS AND OBJECTIVES}

In Japan, multidisciplinary $\mathrm{HF}$ management remains underdeveloped and there are few data regarding telemonitoring for $\mathrm{HF}$ management. Although Japan has a unique universal health insurance system, home telemonitoring for HF management has not been covered by the system except telemonitoring using implantable devices. Therefore, we aimed to investigate whether an HF management programme using a telemonitoring system consisting of automated physiological monitoring devices could reduce mortality and hospital readmission for acute decompensated HF among patients recently hospitalised for HF under the Japanese healthcare system.

Swedberg et al, in their correspondence to the authors of the Tele-HF study, suggested that patient-centred care (PCC) would increase the effectiveness of telemonitoring. They emphasised that telemonitoring needs to focus on patients' self-care instead of reporting data. ${ }^{20} 21$ Therefore, we introduced the concept of PCC into the telemonitoring system used in the present study in order to motivate the patients assigned to the telemonitoring group to maintain adherence to daily measurement of body weight and blood pressure, to enhance clinicianpatient communication and to empower patients in their self-management.

\section{METHODS AND ANALYSIS \\ Study patients}

Patients aged 20 or older with New York Heart Association functional classes II-III who are discharged or scheduled to be discharged following admission for acute HF or acute decompensated chronic HF within 30 days of enrolment into the study are eligible for this study (box 1). The exclusion criteria are as follows: patients with an implantable device (ie, pacemaker, ICD), because an alternating-current signal travels through the body when the patients measure their body weight and body fat using an electronic scale; patients undergoing dialysis or those with a serum creatine level $\geq 3.0 \mathrm{mg} / \mathrm{dl}$; patients with severe liver dysfunction; patients with planned percutaneous coronary intervention or coronary artery bypass grafting; patients unable to stand on a scale safely; patients with a limited life

\section{Box 1 Inclusion criteria}

Scheduled to discharge or discharged from an admission for acute heart failure (HF) or acute decompensated chronic HF within 30 days.

Age $\geq 20$ years

New York Heart Association functional classes II-III. 


\section{Box 2 Exclusion criteria}

- Patients with an implantable device (ie, pacemaker, implantable cardioverter defibrillator), because an alternating-current signal travels through the body when the patients measure their body weight and body fat using an electronic scale.

- Undergoing dialysis or serum creatine level $\geq 3.0 \mathrm{mg} / \mathrm{dl}$.

- Severe liver dysfunction.

- Planned percutaneous coronary intervention or coronary artery bypass grafting.

- Unable to stand on a scale safely.

- Limited life expectancy (malignancy or other cause).

- Severe depression (eg, Patient Health Questionnaire score $\geq 20$ ).

Severe dementia.

Pregnancy.

Without access to a telephone line.

expectancy due to malignancy or other cause; patients in whom severe depression is highly suspected (eg, PHQ-9 $\geq 20$ ); patients with severe dementia; in pregnancy; and patients without access to a telephone line (box 2). Patients suspected of having mild-to-moderate depression (eg, PHQ score: 5-19) are recommended to receive adequate intervention from a psychiatrist or clinical psychologist.

\section{Study design}

The Home Telemonitoring Study for Japanese Patients with Heart Failure (HOMES-HF) is a multicentre, prospective RCT, funded by the Japanese Ministry of Health, Labor and Welfare (Clinical Trials registration number UMIN000006839; http://www.umin.ac.jp/ctr/index. $\mathrm{htm}$ ) and conducted to compare automated physiological data monitoring with usual care. Written informed consent will be obtained by the patient's physician prior to discharge or within 30 days of hospital discharge after admission for acute HF or acute exacerbation of HF. Eligible patients are randomly assigned via a website to either the telemonitoring group or the usual care group by using a minimisation method with biased-coin assignment balancing on age ( $\geq 65$ vs $<65$ years), left-ventricular ejection fraction (LVEF) ( $\geq 30 \%$ vs $<30 \%$ ), and having a history of ischaemic heart disease (IHD; IHD vs non-IHD). The patients and treating physicians are not masked to the treatments, while assessment of the outcome is masked. According to the study protocol, participants will be enrolled until August 2013 and followed until August 2014.

\section{Endpoints}

The primary endpoint is a composite of all-cause death and rehospitalisation due to worsening HF. The secondary endpoints are: all-cause death; cardiac death; allcause rehospitalisation; rehospitalisation due to a cardiovascular cause; rehospitalisation due to worsening HF; worsening of symptoms; cost of medical care; worsening of LVEF or the levels of N-terminal pro B-type natriuretic peptide, high-sensitivity $\mathrm{C}$ reactive protein, pentraxin-3 (PTX3), high-sensitivity cardiac troponin $\mathrm{T}$ or highmolecular weight adiponectin; changes in the Mini Mental State Examination (MMSE) score, the General Self Efficacy Scale (GSES), the Minnesota Living With Heart Failure (MLWHF) score or the PHQ-9 score and adherence to medication.

\section{Telemonitoring system}

The telemonitoring system of the HOMES-HF study consists of an electronic scale, a sphygmomanometer and a device that receives acquired physiological data (blood pressure, pulse rate and body weight) wirelessly and transmits the data to the central web server via the internet. It is commercially available as a health-maintenance product (Karada Karte Tanita health-link Co. Ltd, Tokyo, Japan). These devices are distributed to the participants assigned to the telemonitoring group when they are discharged from the hospital. Patients' physicians encourage the participants assigned to the telemonitoring group, when they demonstrate how to use the monitoring devices after obtaining the informed consent, to measure their body weight and blood pressure by themselves at least once a day at approximately the same time in order to minimise daily variance caused by meals, micturition and bowel movement. The acquired physiological data are automatically transmitted to a central web server immediately after measurement. The telemonitoring centre was newly established at Saga University for the present study, and full-time nurses monitor the acquired data on the secure website 7 days a week (see online supplementary appendix 1). At first contact with the participant by telephone, the monitoring nurses establish communication connection between the monitoring devices and the central web server and arrange a time zone convenient to the participant for regular measuring. Before telemonitoring is started, the patient's physician determines an acceptable range of body weight, blood pressure and pulse rate for each patient and makes a declaration of these ranges to the telemonitoring centre. If the body weight, blood pressure or heart rate would exceed the acceptable range, the monitoring nurses serve a notice to the patient's physician. There are no restrictions on the ability of the patient's physician to perform any interventions in response to the notice, such as providing telephone guidance, changing or adding medications and ordering hospital readmission, with the exception that the physician must provide feedback regarding their interventions to the telemonitoing centre. The patient's physician assumes responsibility for acting on the information.

Introducing the concept of PCC into the telemonitoring system to encourage adherence in participants

After hospital discharge, the patients and their family, especially among elderly persons, have a tendency to be 
socially isolated, and that makes it difficult to practice self-management. In order to motivate the patients assigned to the telemonitoring group to maintain adherence to daily measurement of their body weight and blood pressure, the concept of PCG was proactively introduced into the telemonitoring system for the HOMES-HF study. Enhanced clinician-patient communication, patient empowerment and self-management are the elements of PCC. ${ }^{22}{ }^{23}$ To this end, professionals (typically nurses, although sometimes the patients' physicians) provide advice and education to the patients assigned to the telemonitoring group and create a care plan until the next visit referring to the patients' electronic health records acquired by daily monitoring on the website using a tablet computer in collaboration with the patients at every visit of theirs to the outpatient clinic. According to the protocol, the patients' physicians or nurses have to report to the monitoring centre what they performed for the patient according to the notice from the monitoring nurses. Moreover, we designed the monitoring system to be accessible to the patients' family in order for them to watch over their parents, spouse, siblings or relatives. In this way, we intend to enable the patients to recognise that all healthcare professionals around them and their family are not only monitoring on the website, but also communicating with each other. We believe that these efforts may help reassure patients and their family, as well as encourage them to participate in decision-making on their own treatment by collaborating with healthcare professionals and improve adherence to medical treatment.

\section{Usual care}

Patients assigned to the usual care group are treated by their physician in accordance with the Japanese Circulation Society Guidelines for treatment of chronic HF 2010. Clinicians provide discharge education and encourage the patients to measure their body weight by themselves every day.

\section{Sample size calculation}

We assumed that the HR of the primary endpoint (allcause death and hospitalisation for worsening HF) of the telemonitoring group to the control group would be 0.60 and that the cumulative annual event rate in the usual care group would be 0.30 , based on the result of previous studies. ${ }^{10} 13$ This trial is designed to have $80 \%$ power to detect a $40 \%$ relative reduction in the risk of the primary outcome in the telemonitoring group within 12 months, as compared with the control group, based on an expected death rate at 12 months of $30 \%$ in the control group using a log-rank test with a two-sided $\alpha$ of 0.05 . A total sample size of 420 patients is planned according to the Schoenfeld and Richter method, ${ }^{24}$ with a 2-year period for patient enrolment and a follow-up period of 1 year.

\section{Statistical analysis}

All statistical analyses will be independently performed at the Chiba University Hospital Clinical Research Center (see online supplementary appendix 2). The analyses of the adjudicated primary and secondary outcomes will be conducted using data for all patients who had undergone randomisation, according to the intention-to-treat principle. For the baseline variables, summary statistics will be constructed employing frequencies and proportions for categorical data and means and SD for continuous variables. The patient characteristics will be compared using Fisher's exact test for categorical outcomes and tests for continuous variables, as appropriate. The primary endpoint of a composite of all-cause death and rehospitalisation for worsening $\mathrm{HF}$ will be analysed using the stratified log-rank test for eligible patients with age ( $\geq 65$ vs $<65$ years), LVEF ( $\geq 30 \%$ vs $<30 \%$ ) and history of ischaemic heart disease (IHD vs non-IHD) as stratification factors. Time to events will be estimated using the Kaplan-Meier method, and HRs and 95\% CIs will be calculated using the Cox proportional hazards models with stratification factors. Sensitivity analyses will also be performed by means of the unadjusted Cox models.

All comparisons are planned, and all $p$ values will be two-sided. A $p$ value of less than 0.05 will be considered to be statistically significant. All statistical analyses will be performed using SAS software V.9.3 (SAS Institute, Cary, North Carolina, USA).

\section{Data collection schedule}

At the time each patient is enrolled into the study, investigators at each local site (see online supplementary appendix 6) perform a baseline history and physical examination and conduct a survey of the baseline scores of three types of questionnaires (PHQ-9, MMSE and GSES). The outcomes are assessed at 6 and 12 months after enrolment into the study. Clinicians at each local site submit a report to the data centre at these time points to assess psychosocial status, self-care skills, quality of life and rehospitalisations. We will evaluate the costeffectiveness of the telemonitoring interventions, incorporating the costs associated with hospitalisations, outpatient visits, emergency department visits and home care services.

\section{Study management}

Data on the primary and secondary endpoints and adverse events are collected when the events occur. All data are collected by the independent data management centre established for the present study at the Chiba University Hospital Clinical Research Center (see online supplementary appendix 2). There will be no direct communication between HOMES-HF investigators and the Coordinating Data Center. The clinical data entry (double data entry), cording, data management and reporting will be performed by a data management system, HITCANDIS/DM (HITachi Computer Assisted 
New Drug Information System/Data Management for clinical trial, Hitachi, Ltd Tokyo, Japan). Trained coding specialists will code the clinical data using standard coding dictionaries including MedDRA for adverse events and medical history and considering WHO-DD for concomitant medications. All the data management processes are tracked electronically, allowing regular updates on patient status, data receipt including missing segments or pages, data entry and verification, data query status and protocol deviations. In order to ensure consistency, integrity and accuracy for this study, these processes are based on the standard operating procedures.

An independent endpoint committee (see online supplementary appendix 3) consisting of three members, who are blinded to any information relating to the group allocations, evaluates each event and classifies the results. An independent data and safety monitoring board (see online supplementary appendix 4) composed of three members reviews all reports from the endpoint committee to advise early termination of the study for safety, scientific or ethical reasons. A steering committee (see online supplementary appendix 5) is responsible for the study design and scientific execution of the study.

\section{Laboratory measurements}

The plasma PTX3 levels are measured with a sandwich ELISA kit (Perseus Proteomics Inc, Tokyo, Japan) based on a previously described method. ${ }^{25}$ The plasma HMW-adiponectin levels are measured using a sandwich ELISA kit (Fujirebio, Tokyo, Japan) based on a monoclonal antibody to human HMW-adiponectin, IH7. ${ }^{26}$

\section{Author affiliations}

${ }^{1}$ Department of Cardiovascular Medicine, Saga University, Saga, Japan ${ }^{2}$ Chiba University Hospital Clinical Research Center, Chiba University, Chiba, Japan

${ }^{3}$ Division of Cardiolovascular Medicine, Department of Molecular Medicine and Therapeutics, Tottori University, Yonago, Japan

${ }^{4}$ Department of Cardiovascular Medicine, Tohoku University Graduate School of Medicine, Sendai, Japan

${ }^{5}$ Department of Cardiology, St. Luke's International Hospital, Tokyo, Japan

${ }^{6}$ Department of Cardiology, Saga Prefectural Hospital Koseikan, Saga, Japan

${ }^{7}$ Division of Cardiovascular Medicine, Saitama Medical Center, Jichi Medical University, Omiya, Japan

${ }^{8}$ Department of Medicine, Division of Cardiovascular Medicine, Jichi Medical University, Shimotsuke, Japan

${ }^{9}$ Department of Cardio-Angiology, Kitasato University School of Medicine, Sagamihara, Japan

${ }^{10}$ Department of Cardiovascular Medicine, Hokkaido University Graduate

School of Medicine, Sapporo, Japan

${ }^{11}$ Cardiovascular Division, Department of Internal Medicine, Hyogo College of Medicine, Nishinomiya, Japan

${ }^{12}$ Department of Cardiovascular Medicine, National Cerebral and

Cardiovascular Center, Suita, Japan

${ }^{13}$ Department of Cardiovascular Medicine, Dokkyo Medical University, Mibu, Japan

${ }^{14}$ Department of Cardiology, Nippon Medical School Chiba-Hokusoh Hospital, Inzai, Japan

Acknowledgements The authors gratefully acknowledge Tomoko Hirotaki, Aya Yamada, Sae Katafuchi, Junko Ishida for their valuable assistance.
Contributors Contributors and details of the study investigators of the HOMES-HF study are described also in the supplemental file (appendices). (1) NK, YS, HH, TI, YF, KY, HT, TM, MK, TI, HS, SM, YS and KN participated in the conception and design. (2) MA, YK, KN, AM, DN, DM, YY and KE participated in conducting the trial and acquisition of data. (3) NK and YS participated in drafting the article or revising it critically for important intellectual content. (4) All authors participated in the final approval of the version to be published.

Funding The HOMES-HF study is supported by grants from the Japanese Ministry of Health, Labor, and Welfare Comprehensive Research on Aging and Health (KR23000003, KR24000001). The funder is the government, which will not have any role in interpreting the results or deciding if the results should be published.

Competing interests All authors have completed the ICMJE form for disclosure of potential conflicts of interest at http://www.icmje.org/ coi_disclosure.pdf (available on request from the corresponding author) and declare that NK is currently an endowed chair from Fukuda Denshi Co., Ltd, which is a medical equipment manufacturer. The company has no relation to the monitoring equipment used in this study. All authors have no other relationships or activities that could appear to have influenced the submitted work.

Ethics approval All participants will provide their written informed consent, and the study protocol has been approved by the institutional review board of Saga University and each participating site.

Provenance and peer review Not commissioned; internally peer reviewed.

Data sharing statement No additional data are available.

\section{REFERENCES}

1. Heart Disease and Stroke Statistics-2007 Update, American Heart Association.

2. Tsutsui H, Tsuchihashi-Makaya M, Kinugawa S. Clinical characteristics and outcomes of heart failure with preserved ejection fraction: lessons from epidemiological studies. J Cardiol 2010;55:13-22.

3. Tsuchihashi M, Tsutsui $\mathrm{H}$, Kodama K, et al. Clinical characteristics and prognosis of hospitalized patients with congestive heart failure a study in Fukuoka, Japan. Jpn Circ J 2000;64:953-9.

4. Tsuchihashi M, Tsutsui $\mathrm{H}$, Kodama K, et al. Medical and socioenvironmental predictors of hospital readmission in patients with congestive heart failure. Am Heart J 2001;142:E7.

5. Okura Y, Ramadan MM, Ohno Y, et al. Impending epidemic: future projection of heart failure in Japan to the year 2055. Circ $J$ 2008;72:489-91.

6. Rich MW, Beckham V, Wittenberg C, et al. A multidisciplinary intervention to prevent the readmission of elderly patients with congestive heart failure. N Engl J Med 1995;333:1190-5.

7. Whellan DJ, Hasselblad V, Peterson E, et al. Metaanalysis and review of heart failure disease management randomized controlled clinical trials. Am Heart J 2005;149:722-9.

8. Holland R, Battersby J, Harvey I, et al. Systematic review of multidisciplinary interventions in heart failure. Heart 2005;91:899-906.

9. Yu DSF, Thompson DR, Lee DTF. Disease management programmes for older people with heart failure: crucial characteristics which improve post-discharge outcomes. Eur Heart $J$ 2006;27:596-612.

10. Chaudhry SI, Phillips AO, Stewart SS, et al. Telemonitoring for patients with chronic heart failure: a systematic review Harlan M. J Cardiac Fail 2007;13:56-62.

11. Benatar D, Bondmass M, Ghitelman J, et al. Outcomes of chronic heart failure. Arch Intern Med 2003;163:347-52.

12. Goldberg LR, Piette JD, Walsh MN, et al. Randomized trial of a daily electronic home monitoring system in patients with advanced heart failure: the weight monitoring in heart failure (WHARF) trial. Am Heart J 2003;146:705-12.

13. Klersy C, De Silvestri A, Gabutti G, et al. A meta-analysis of remote monitoring of heart failure patients. J Am Coll Cardiol 2009;54:1683-94.

14. Inglis SC, Clark RA, McAlister FA, et al. Structured telephone support or telemonitoring programmes for patients with chronic heart failure. Cochrane Database Syst Rev 2010:CD007228.

15. Chaudhry SI, Mattera JA, Curtis JP, et al. Telemonitoring in patients with heart failure. N Engl J Med 2010;363:2301-9. 
16. Koehler F, Winkler S, Schieber M, et al., on behalf of the Telemedical Interventional Monitoring in Heart Failure Investigators. Impact of remote telemedical management on mortality and hospitalizations in Ambulatory patients with chronic heart failure: the telemedical interventional monitoring in Heart Failure Study. Circulation 2011;123:1873-80.

17. Konstam MA. Does home monitoring heart failure care improve patient outcomes? Home monitoring should be the central element in an effective program of heart failure disease management. Circulation 2102;125:820-7.

18. Desai AS. Does home monitoring heart failure care improve patient outcomes? Home monitoring heart failure care does not improve patient outcomes: looking beyond telephone-based disease management. Circulation 2102;125:828-36.

19. Koehler F, Winkler S, Schieber M, et al. Telemedicine in heart failure: Pre-specified and exploratory subgroup analyses from the TIM-HF trial. Int J Cardiol 2012; 161:143-50.

20. Swedberg K, Wolf A, Ekman I. Telemonitoring in patients with heart failure. N Engl J Med 2011;364:1078.
21. Cleland JG, Ekman I. Enlisting the help of the largest health care workforce-patients. JAMA 2010;304:1383-4.

22. Walsh MN, Bove AA, Cross RR, et al. American College of Cardiology Foundation. ACCF 2012 health policy statement on patient-centered care in cardiovascular medicine: a report of the American College of Cardiology Foundation Clinical Quality Committee. J Am Coll Cardiol 2012;59:2125-43.

23. Ekman I, Wolf A, Olsson LE, et al. Effects of person-centred care in patients with chronic heart failure: the PCC-HF study. Eur Heart $J$ 2012;33:1112-19.

24. Schoenfeld DA, Richter JR. Nomograms for calculating the number of patients needed for a clinical trial with survival as an endpoint. Biometrics 1982;38:163-70.

25. Inoue K, Sugiyama A, Reid PC, et al. Establishment of high sensitivity plasma assay for human pentraxin3 as a marker for unstable angina pectolis. Arterioscler Thromb Vasc Biol 2007:27:161-7.

26. Nakano Y, Tajima S, Yoshimi A, et al. A novel enzyme-linked immunosorbent assay specific for high-molecular-weight adiponectin. J Lipid Res 2006;47:1572-82. 